\title{
ACY-1215 accelerates vemurafenib induced cell death of BRAF-mutant melanoma cells via induction of ER stress and inhibition of ERK activation
}

\author{
UEIHUEI PENG ${ }^{1}$, ZHIHAO WANG $^{2}$, SA PEI $^{1}$, YUNCHAO OU ${ }^{1}$, \\ PENGCHAO HU ${ }^{2}$, WANHONG LIU ${ }^{2}$ and JIQUAN SONG ${ }^{1}$ \\ ${ }^{1}$ Department of Dermatology, Zhongnan Hospital of Wuhan University; \\ ${ }^{2}$ School of Basic Medical Sciences, Wuhan University, Wuhan, Hubei 430071, P.R. China
}

Received July 7, 2016; Accepted November 28, 2016

DOI: $10.3892 / o r .2016 .5340$

\begin{abstract}
BRAF}^{\mathrm{V} 600 \mathrm{E}}$ mutation is found in $~ 50 \%$ of melanoma patients and $\mathrm{BRAF}^{\mathrm{V} 600 \mathrm{E}}$ kinase activity inhibitor, vemurafenib, has achieved a remarkable clinical response rate. However, most patients treated with vemurafenib eventually develop resistance. Overcoming primary and secondary resistance to selective BRAF inhibitors remains one of the most critically compelling challenges for these patients. HDAC6 has been shown to confer resistance to chemotherapy in several types of cancer. Few studies focused on the role of HDAC6 in vemurafenib resistance. Here we showed that overexpression of HDAC6 confers resistance to vemurafenib in BRAF-mutant A375 cells. ACY-1215, a selective HDAC6 inhibitor, inhibits the proliferation and induces the apoptosis of A375 cells. Moreover, ACY-1215 sensitizes A375 cells to vemurafenib induced cell proliferation inhibition and apoptosis induction, which occur partly through induction of endoplasmic reticulum (ER) stress and inactivation of extracellular signalregulated kinase (ERK). Taken together, our results suggest that the inhibition of HDAC6 may be a promising strategy for the treatment of melanoma and overcoming resistance to vemurafenib.
\end{abstract}

\section{Introduction}

Melanoma is one of the most aggressive human cancers and its incidence continues to increase worldwide (1). Although melanoma is one of the most aggressive human cancers, the identification of multiple melanoma driven mutations

Correspondence to: Dr Jiquan Song, Department of Dermatology, Zhongnan Hospital of Wuhan University, 169 Donghu Road, Wuhan, Hubei 430071, P.R. China

E-mail: songjiq@126.com

Abbreviations: PARP, poly(ADP-ribose) polymerase; Raf, rapidly accelerated fibrosarcoma

Key words: vemurafenib, melanoma, HDAC6, ER stress, ERK and improved understanding of cancer immune tolerance checkpoints has led to identification of new therapeutic opportunities for patients $(1,2)$. The most common of these mutations is $\mathrm{BRAF}^{\mathrm{V} 600 \mathrm{E}}$. It is found in $50-60 \%$ of melanomas and successful targeting of the $\mathrm{BRAF}^{\mathrm{V} 600 \mathrm{E}}$, such as vemurafenib, has produced amazing clinical responses in patients with melanoma harboring this mutation $(3,4)$. Vemurafenib is the first drug approved for the treatment of BRAF-mutant cancer (3). Unfortunately, the majority of responding patients eventually develop resistance and disease progression, typically within 5-7 months after starting the treatment (5). Therefore, overcoming the acquired resistance remains a considerable therapeutic challenge to achieve durable responses and prolonged survival in these patients.

A variety of molecular mechanisms have been identified to be involved in acquired resistance to vemurafenib (5). Unlike acquired resistance to epidermal growth factor receptor (EGFR) tyrosine kinase inhibitors are mainly generated by secondary mutations in EGFR, multiple mechanisms account for the resistance to vemurafenib (6). These mechanisms include the loss/inactivation of phosphatase and tensin homolog (PTEN) function, deletion of the retinoblastoma protein (RB), elevated expression of the kinases CRAF, amplification of cyclin D1, alternative splicing of BRAF mRNA, activating mutations in NRAS, mitogen-activated protein kinase kinase (MEK), or AKT and persistent activation of receptor tyrosine kinases, including EGFR, IGF1R and platelet-derived growth factor $\beta$ (PDGFR $\beta$ ) (7). The diversity of resistance to vemurafenib implies that BRAF inhibitor resistance can be overcome through broadly targeted strategies that inhibit multiple pathways simultaneously.

Histone deacetylases (HDACs) are a group of enzymes that function by catalyzing the removal of the acetyl groups of both histones and non-histone proteins (8). HDACs are considered to be among the most promising targets in drug development for cancer therapy (8). Among the 18 HDACs, HDAC6 has recently sparked great interest as it deacetylates various substrates involved in the regulation of protein trafficking and degradation, autophagy, apoptosis, cell cycle, migration and proliferation (9). HDAC6 is required for the proliferation and metastasis of melanoma cells and knockdown of HDAC6 
decreases proliferation and induces cell cycle arrest of melanoma cells $(10,11)$. HDAC6 has also been shown to confer resistance to chemotherapy in many types of cancer (12-14). Many pathways involved in acquired resistance to vemurafenib are known to be regulated by HDAC6 $(7,15)$. However, the role of HDAC6 in vemurafenib resistance is still unclear.

Here we report that overexpression of HDAC6 confers resistance to vemurafenib in the $\mathrm{BRAF}^{\mathrm{V} 600 \mathrm{E}}$ melanoma cell line A375. HDAC6 deacetylase activity selective inhibitor, ACY-1215, impairs proliferation and induces apoptosis of A375. Combination use of vemurafenib with ACY-1215 displayed an additive therapeutic effect in BRAF-mutant melanoma cells by inducing ER stress and inactivation of extracellular signalregulated kinase (ERK). Our results suggest that inhibition of HDAC6 may be a promising strategy for overcoming the resistance to vemurafenib in melanoma harboring $\mathrm{BRAF}^{\mathrm{V} 600 \mathrm{E}}$ mutation.

\section{Materials and methods}

Cell culture. The BRAF-mutant melanoma cell line A375 was obtained from the American Type Culture Collection (Manassas, VA, USA). A375 was cultured in RPMI-1640 medium (HyClone) supplemented with $10 \%$ FBS and $1 \%$ penicillin/streptomycin.

Reagents. ACY-1215 and Z-VAD-FMK were purchased from Medchem Express (Monmouth Junction, NJ, USA). The anti-PARP (\#9542), anti-eIF2 $\alpha$ (\#5324), anti-p-eIF2 $\alpha$ (\#9721) and anti-CHOP (\#2895) antibodies were obtained from Cell Signaling Technology (Danvers, MA, USA). The anti-caspase-3 (EAP0893) antibody was from Elabscience (Wuhan, Hubei, China). The anti-p-ERK (ab76299) antibody was purchased from Abcam (Cambridge, UK). The anti-ERK (16443-1-AP) and anti-GFP (50430-2-AP) antibodies was obtained from Proteintech (Chicago, IL, USA). The anti- $\beta$-actin mouse monoclonal antibody (AM1021B) was purchased from Abgent (San Diego, CA, USA).

Cell proliferation and colony formation detection. A375 cell proliferation was measured at the indicated times by Cell counting kit-8 (CCK-8) kit (Dojindo, Kumamoto, Japan Japan). A375 colony formation was measured by seeding cells in 6-well plates 1,000 per well and were cultured over a 14-day period. Colonies were fixed in $4 \%$ paraformaldehyde and stained with $0.1 \%$ crystal violet.

Annexin V assay of cell apoptosis. Effects of plasmids or drugs on apoptosis of A375 cells were evaluated by Annexin V/ 7-aminoactinomycin D (7-AAD) assay. Flow cytometric analysis of A375 cells labeled with Annexin V-phycoerythrin (PE) and 7-AAD apoptosis detection kit (BD Biosciences, San Jose, CA, USA) was performed according to the manufacturer's instructions. The rates of cellular apoptosis were acquired immediately on a FACSArial flow cytometer (BD Biosciences).

Plasmids and transfection. The plasmid encoding HDAC6 was kindly provided by Professor Jun Zhou (16). The plasmids have been validated by sequencing, transfection and subsequent western blotting. The plasmids were transfected into cells with Lipofectamine 2000 transfection reagent (Invitrogen, Waltham, MA, USA) according to the manufacturer's protocol.

Protein extraction and western blotting. A375 cells were lysed in RIPA lysis buffer (1\% Triton X-100, $150 \mathrm{mM} \mathrm{NaCl}, 1 \mathrm{mM}$ EDTA, $1 \%$ NP-40, $0.1 \%$ SDS, $0.5 \%$ sodium deoxycholate and $50 \mathrm{mM}$ Tris- $\mathrm{HCl}, \mathrm{pH}$ 7.4) with protease inhibitor cocktails and phosphatase inhibitor cocktails from Roche (Mannheim, Germany) on ice for $30 \mathrm{~min}$ and centrifuged at 12,000 rpm for $15 \mathrm{~min}$ to collect whole cell lysate. Protein extracts were subjected to electrophoresis on a 10\% SDS-PAGE gel and transferred onto PVDF membrane (Roche). Then the membrane was then incubated in blocking buffer for $2 \mathrm{~h}$ before the addition of the primary antibodies. The secondary antibody used was the horseradish peroxidase-conjugated goat anti-rabbit/mouse secondary antibody (Proteintech). Signals were detected using WesternBright ECL HRP substrate (Advansta, Menlo Park, CA, USA) and developed with Kodak film.

Statistical analysis. Statistical analysis was performed with Student's t-test. All analyses were realized by using the statistical software SPSS19.0 (IBM Corp., Armonk, NY, USA). Data are expressed as the mean \pm standard deviation (SD). $\mathrm{P}<0.05$ was considered a statistically significant difference.

\section{Results}

HDAC6 confers resistance to vemurafenib mediated killing of BRAF-mutant A375 cells. HDAC6 can confer resistance to chemotherapy in several types of cancer (12-14), but the role of HDAC6 in melanoma chemotherapy resistance is largely unknown. HDAC6 has been shown to be overexpressed in melanoma cell lines and tissues (10). We speculate that overexpression of HDAC6 in melanoma might confer resistance to vemurafenib. As shown in Fig. 1A, overexpression of HDAC6 significantly improved the percentage of viable A375 cells after vemurafenib treatment. We also came to the same conclusion by the colony formation assay (Fig. 1B). Vemurafenib has been shown to cause apoptotic cell death at elevated concentrations, thus we investigated whether HDAC6 regulates vemurafenib mediated cell apoptosis in melanoma cells. Overexpression of HDAC6 significantly decreased the percentage of apoptotic cells after vemurafenib treatment (Fig. 1C). A decrease in apoptosis was further evidenced by detection of cleaved PARP and caspase-3 (Fig. 1D).

ACY-1215, a selective HDAC6 inhibitor, sensitizes A375 cells to vemurafenib. Because HDAC6 confers resistance to vemurafenib, we speculated that the inhibition of HDAC6 might contribute to an increase in the efficiency of vemurafenib in melanoma. To further assess this possibility, we tested the effect of HDAC6 inhibition on A375 using the selective inhibitor ACY-1215. ACY-1215 is the first oral, selective HDAC6 inhibitor in clinical trials and was well tolerated as monotherapy $\leq 360 \mathrm{mg} /$ day $(17,18)$. Unlike non-selective HDAC inhibitors, which are associated with severe fatigue, vomiting, diarrhea and myelosuppression (18). ACY-1215 offers a therapeutic advantage due to minimal toxicity. 
A

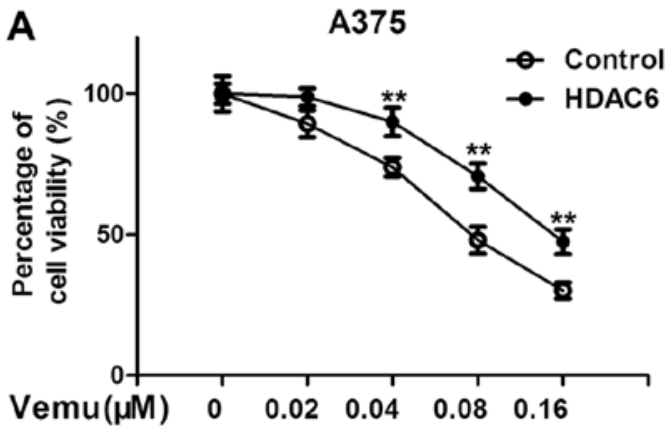

C

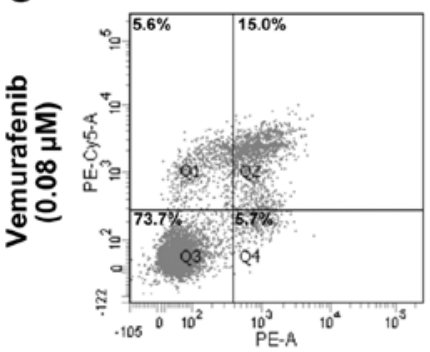

B

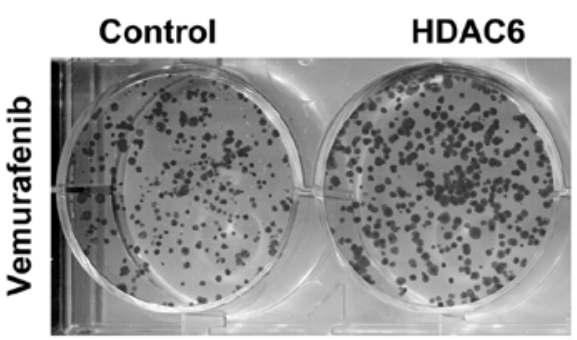

A375

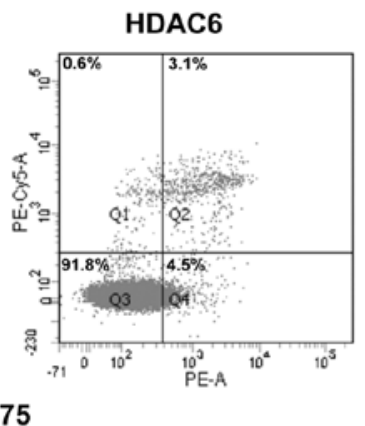

D

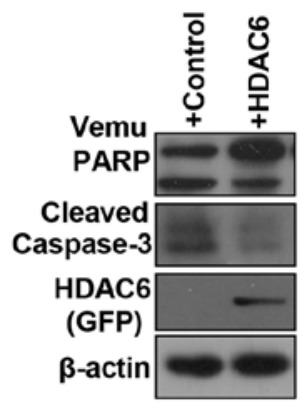

Figure 1. Upregulation of HDAC6 confers resistance to vemurafenib-mediated cell proliferation inhibition. (A) A375 cells were transfected with indicated plasmids and treated with the indicated concentrations of vemurafenib. Cell viability was measured by CCK-8 assay. (B) The effect of HDAC6 overexpression on cell clone formation was measured by plate clone formation assay. (C and D) A375 cells were transfected with indicated plasmids and treated with vemurafenib. After $72 \mathrm{~h}$, Annexin V-PE staining (C) assay and western blotting (D) assay were utilized to detect apoptosis. ${ }^{* *} \mathrm{P}<0.01, \mathrm{t}$-test.
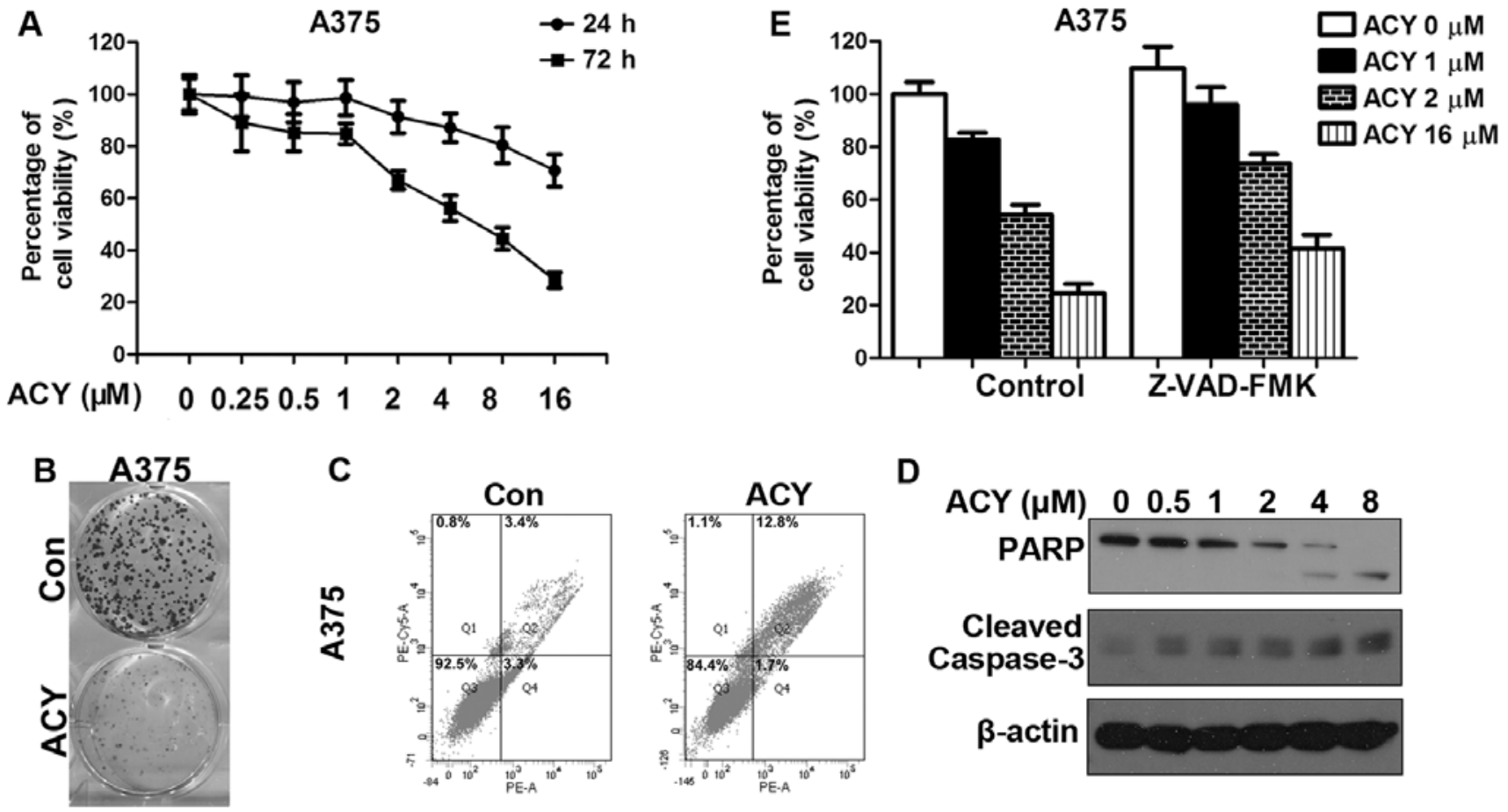

D

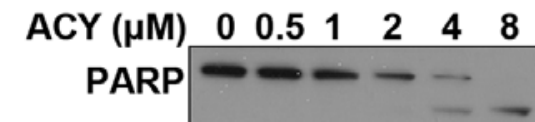

\section{Cleaved \\ Caspase-3}

\section{$\beta$-actin}

Figure 2. Inhibition of HDAC6 by ACY-1215 inhibits the proliferation and induces apoptosis of A375 cells. (A) Curves show the concentration-dependent effect of ACY-1215 on the viability of A375. (B) The effect of ACY-1215 on cell clone formation was measured by plate clone formation assay. (C and D) A375 cells were treated with indicated concentrations of ACY-1215. After $72 \mathrm{~h}$, Annexin V-PE staining (C) assay and western blotting (D) assay were utilized to detect apoptosis. (E) The role of apoptosis in ACY-1215 induced cell proliferation inhibition.

HDAC6 is required for the proliferation and metastasis of melanoma cells $(10,11)$. However, few studies have focused on the function of ACY-1215 in melanoma cells. We first assessed growth inhibition in response to ACY-1215 treatment in A375. A dose-dependent decrease in cell proliferation was observed (Fig. 2A). The same conclusion was drawn from the results of 

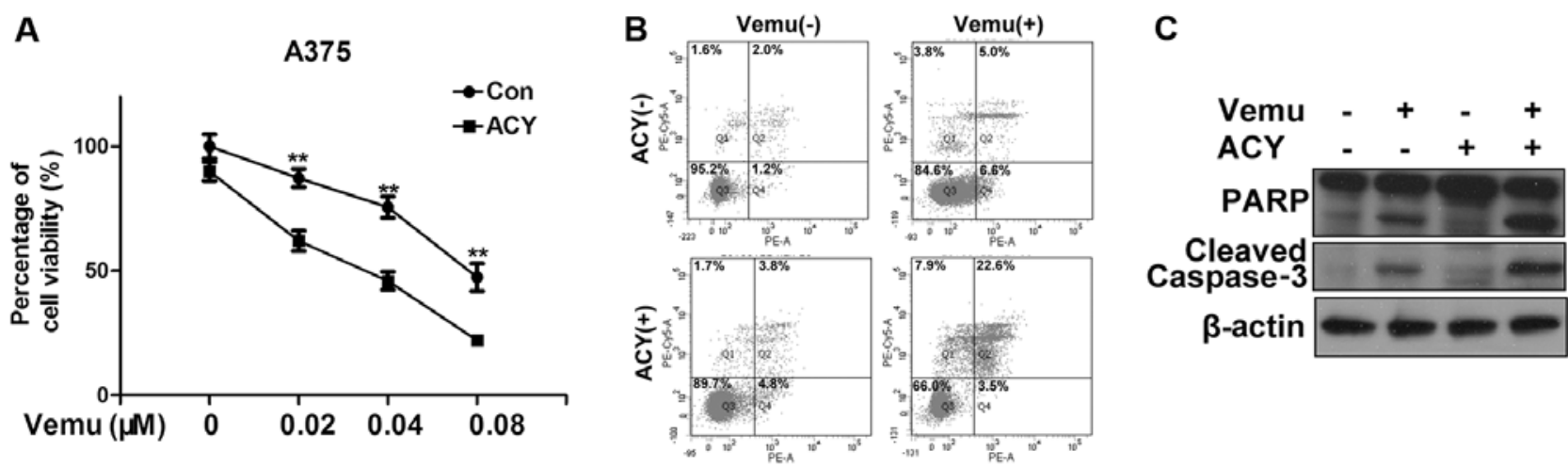

Figure 3. ACY-1215 sensitizes A375 cells to vemurafenib. (A) A375 cells were treated with the given concentrations of vemurafenib (Vemu) and $0.5 \mu \mathrm{M}$ ACY-1215 (ACY). CCK-8 assay was utilized to measure cell viability. (B and C) A375 cells were treated with $0.04 \mu \mathrm{M}$ vemurafenib with or without ACY-1215. After 72 h, Annexin V-PE staining (B) assay and western blotting (C) assay were utilized to detect apoptosis.
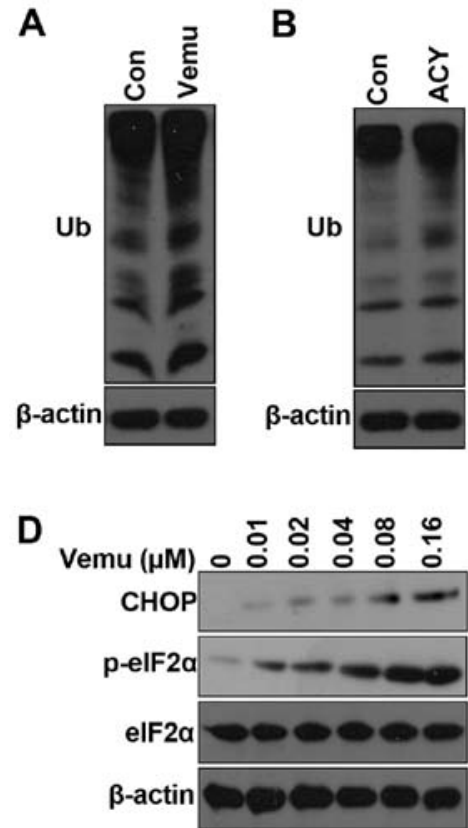

B

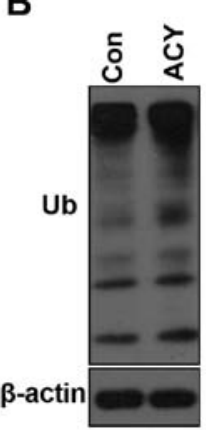

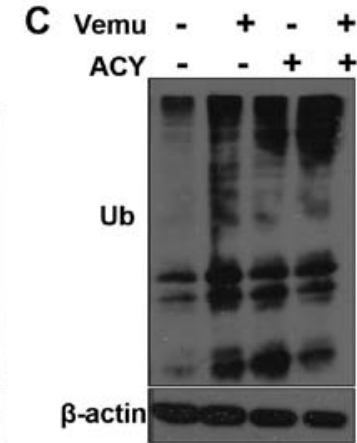
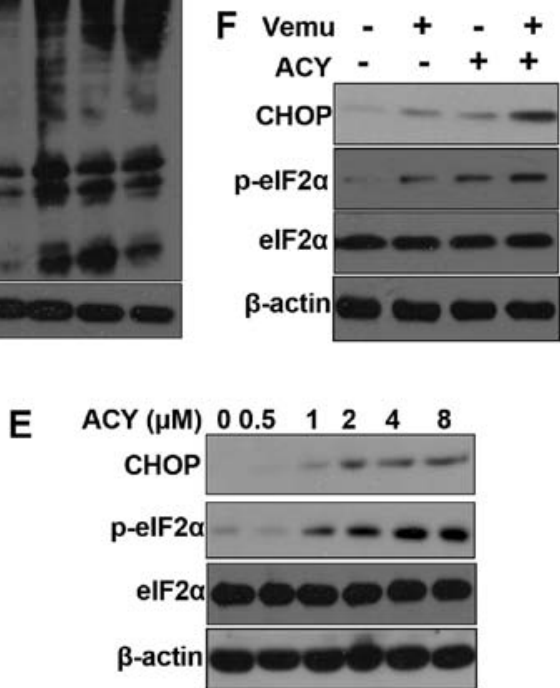

Figure 4. ACY-1215 promotes vemurafenib-induced ER stress. (A, B, D ans E) A375 cells were treated with the vemurafenib (Vemu) (A and D) or ACY-1215 ( $\mathrm{B}$ and $\mathrm{E}$ ) and cells were harvested for western blot analysis. (C and F) A375 cells were treated with $0.04 \mu \mathrm{M}$ vemurafenib with or without $0.5 \mu \mathrm{M}$ ACY-1215. After $48 \mathrm{~h}$, cells were harvested for western blot analysis.

the clone formation assay. We noted a significant decrease in clone numbers after ACY-1215 treatment (Fig. 2B). ACY-1215 also induces apoptosis in A375, as evidenced by increases in the number of Annexin V-positive cells and the cleavage of PARP and caspase-3 (Fig. 2C and E). Combination inhibition of apoptosis by pan caspase inhibitor Z-VAD-FMK with ACY-1215 remarkablely improved the percentage of cell viability compared to ACY-1215 alone (Fig. 2D). To examine whether a cooperative effect exists between ACY-1215 and vemurafenib in the chemotherapeutic treatment of melanoma, we treated A375 cells with ACY-1215 and vemurafenib either alone or in combination. In agreement with our hypothesis, the co-treatment of ACY-1215 and vemurafenib significantly reduced the cell viability compared to vemurafenib alone (Fig. 3A). We also observed a significant increase of the percentage of apoptotic cells after cotreatment of vemurafenib with ACY-1215 compared to vemurafenib alone (Fig. 3B). The protein levels of cleaved PARP and caspase- 3 also increased after co-treatment (Fig. 3C).

ACY-1215-induced ER stress plays a role in overcoming acquired resistance to vemurafenib. Next, we investigated the mechanism by which ACY-1215 contribute to the vemurafenibinduced cell death of melanoma cells. HDAC6 plays a vital role in regulation of degrading misfolded protein through the ubiquitin proteasome system and autophagy $(19,20)$. We found that both vemurafenib and ACY-1215 increased the accumulation of polyubiquitinated proteins (Fig. 4A and B). Moreover, the combination of ACY-1215 plus vemurafenib increased the accumulation of polyubiquitinated proteins compared with either agent alone (Fig. 4C). Accumulation of polyubiquitinated proteins can induce ER stress (21). Vemurafenib has been 


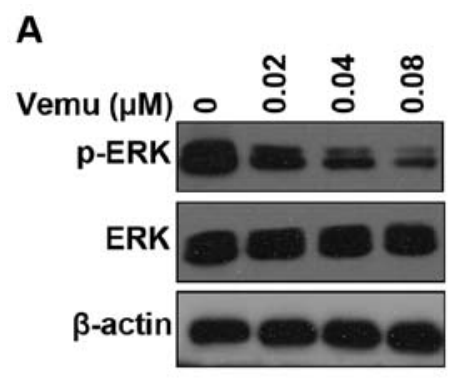

B GFP

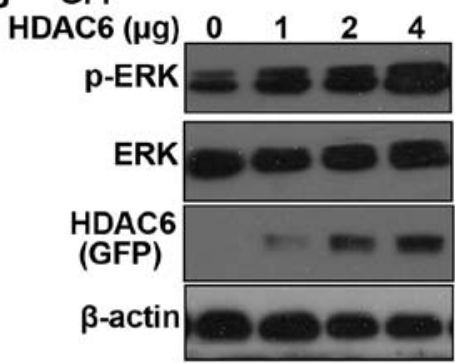

C

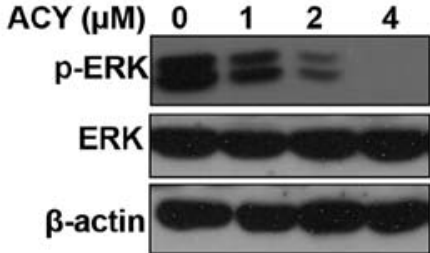

D
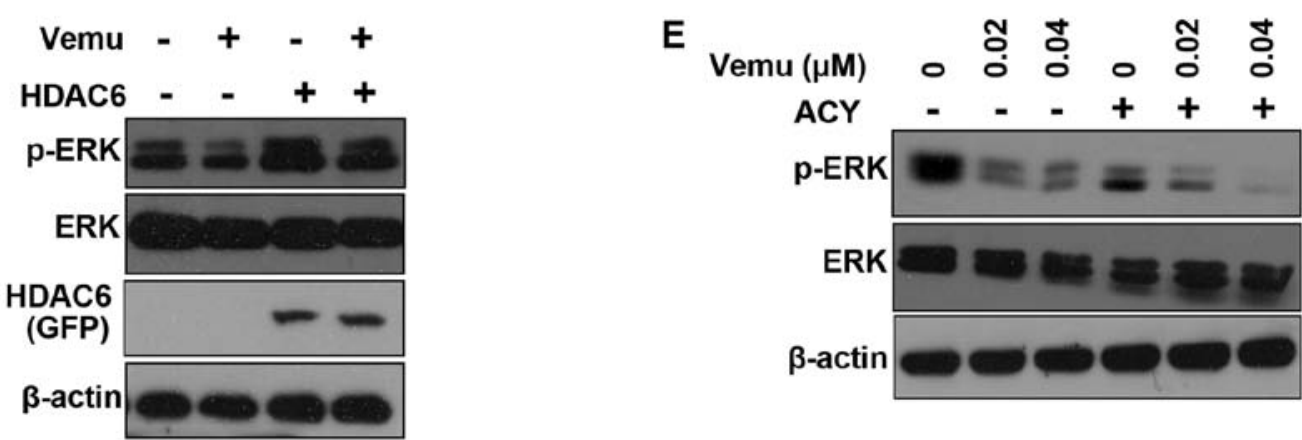

Figure 5. HDAC6 promotes the activation of ERK and ACY-1215 inactivates ERK. (A and C) A375 cells were treated with indicated concentrations of vemurafenib (A) or ACY-1215 (C) and then cells were harvested for western blot analysis. (B) A375 cells were transfected with indicated plasmids and then cells were harvested for western blot analysis. (D) A375 cells were transfected with indicated plasmids and treated with or without vemurafenib, cells were harvested for western blot analysis. (E) A375 cells were treated with vemurafenib with or without ACY-1215. Cell lysis was subjected to subsequent western blotting.

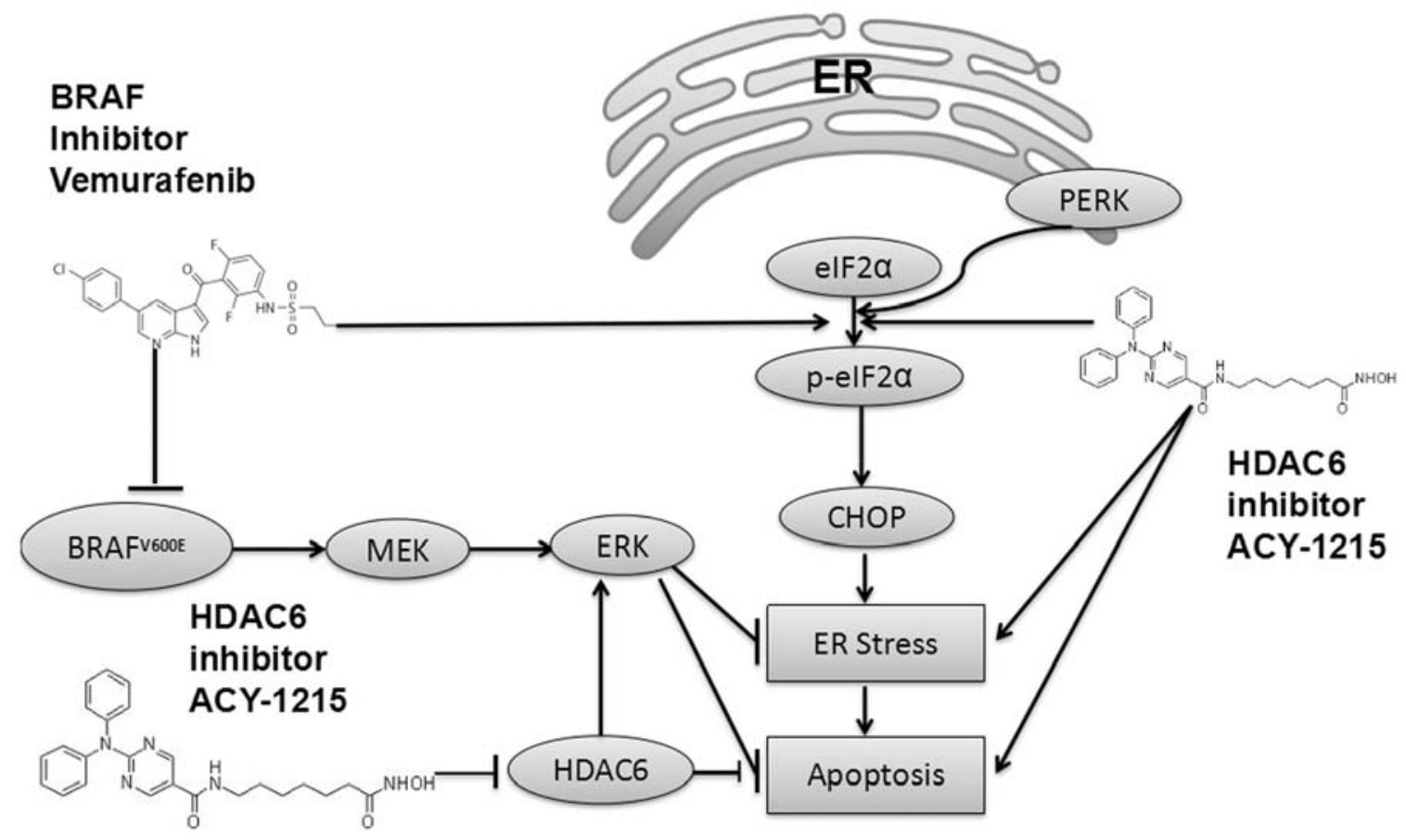

Figure 6. Illustration of how HDAC6 inhibitor ACY-1215 sensitizes BRAF-mutant melanoma cells to vemurafenib.

shown to induce endoplasmic reticulum (ER) stress-mediated apoptosis to kill BRAF ${ }^{\mathrm{V} 600 \mathrm{E}}$ melanoma cells (22). Upon ER stress, the sensor PERK is activated and phosphorylates eIF2a $(23,24)$. During ER stress, the protein levels of CHOP are also elevated and CHOP functions to mediate programmed cell death $(24,25)$. To determine the onset of ER stress in response to ACY-1215, we examined the phosphorylation of eIF $2 \alpha$ by western blotting and found that it was rapidly phosphorylated after ACY-1215 treatment (Fig. 4D). Vemurafenib can also clearly induce ER stress as previously reported (Fig. 4C). Combination treatment of vemurafenib and ACY-1215 significantly increased the phosphorylation of eIF $2 \alpha$ and protein 
levels of CHOP. Taken together, these results indicate that ACY-1215 induced ER stress plays a role in overcoming resistance to vemurafenib.

HDAC6-mediated ERK activation partly accounts for its oncogenic role in melanoma. Hyperactivation of the RAF-MEK-ERK signaling pathway plays a vital role in tumorigenesis of BRAF-mutant melanoma (26). RAF kinase directly phosphorylates MEK, which in turn phosphorylates ERK to promote cell proliferation and to inhibit apoptosis (27). Vemurafenib blocks ERK phosphorylation to inhibit proliferation of BRAF-mutant cells $(3,22)$, this was also confirmed in our experiment (Fig. 5A). HDAC6 can induce ERK hyperactivation in Madin-Darby canine kidney (MDCK) and HEK293T cells (28). Overexpression of HDAC6 also increased ERK activity in A375 cells (Fig. 5B). Conversely, ACY-1215 decreases the phosphorylation of ERK in a dose-dependent manner (Fig. 5C). Moreover, overexpression of HDAC6 increased ERK activity after vemurafenib treatment (Fig. 5D). We found that the activation of ERK was inhibited by the combination of ACY-1215 and vemurafenib compared with the administration of either inhibitor alone (Fig. 5E). Taken together, our results show that inhibition of HDAC6 sensitizes A375 cells to vemurafenib partly via inactivation of ERK.

\section{Discussion}

In this study, we addressed whether inhibition of HDAC6 can sensitize BRAF-mutant melanoma cells to vemurafenib (PLX4032). Overexpression of HDAC6 confers resistance to vemurafenib in A375 cells. Inhibition of HDAC6 by a selective inhibitor, ACY-1215, sensitizes A375 cells to vemurafenib mediated proliferation inhibition and apoptosis induction. Moreover, ACY-1215 mediated ER stress induction and ERK inactivation might play a role in overcoming acquired resistance to vemurafenib.

Immune checkpoint blockade by directly targeting programmed death 1 (PD-1) with monoclonal antibodies has gained great success in clinic in patients with advanced melanoma $(29,30)$. PD-1 can negatively regulate the effector phase of T-cell responses after ligation of PD-1 ligand 1 (PD-L1), which plays a vital role in the ability of tumor cells to evade the immune system (31). Therefore, cancer tissues can limit the host immune response via upregulation of PD-1 ligand (PD-L1) and its ligation to PD-1 on antigen-specific $\mathrm{CD}^{+}$cells (31). However, not all patients respond equally to this treatment (32). There is an urgent need to identify new potential therapeutic adjuvants to improve immunotherapeutic efficiency towards melanoma (33). Some HDACs have been shown to function as modulators of the immune response (33). Among them, HDAC6 recently sparked great interest as the inhibition of HDAC6 can downregulate the expression of PD-L1 in primary melanoma samples and cell lines (33). Woan et al also showed that targeting HDAC6 can enhance antitumor immunity in melanoma cells (11). Therefore, HDAC6 might also exert oncogenic functions by mediating immune escape in melanoma. Combination use of HDAC6 inhibitors and PD1 inhibitors might further benefit melanoma patients. HDAC6 inhibitors are being tested in clinical trials in combination with nivolumab (PD1 blocking antibody) in patients with unresectable non-small cell lung cancer (NSCLC) (https://www.clinicaltrials.gov/, ClinicalTrials.gov Identifier:NCT02635061).

Prahallad et al reported that feedback activation of EGFR mediated by $\mathrm{BRAF}^{\mathrm{V} 600 \mathrm{E}}$ inhibition might contribute to unresponsiveness of colon cancer to vemurafenib (34). Corcoran et al proved that EGFR-mediated reactivation of MAPK signaling contributes to resistance to vemurafenib in colorectal cancers (35). HDAC6 regulates EGFR endocytic trafficking and degradation $(36,37)$. HDAC6 has been shown to confer resistance to chemotherapy through stabilization and activation of EGFR $(13,38,39)$. Therefore, HDAC6 might also confer resistance to vemurafenib via regulation of EGFR stabilization and activation. Pharmacologic inhibition of heat shock protein 90 (HSP90) can abrogate both acquired and intrinsic vemurafenib resistance by restoring apoptotic response (7). Many proteins involved in vemurafenib resistance, including mutated BRAF, CRAF, cyclin D1, IGF1R, AKT and CDK4 are known to be regulated by HSP90 (7). HDAC6 can directly regulate HSP90 chaperone activity through deacetylation of HSP90 (15). Therefore, HDAC6 can also confer resistance to vemurafenib via regulation of HSP90 activity. Autophagy inhibition improves chemosensitivity in $\mathrm{BRAF}^{\mathrm{V} 600 \mathrm{E}}$ tumors (40). HDAC6 regulates autophagosome maturation and inhibition of HDAC6 has been shown to sensitize cancer cells to chemotherapy by inhibiting autophagy (20).

In conclusion, we found that HDAC6 confers resistance to vemurafenib in BRAF-mutant melanoma and that inhibition of HDAC6 impairs the proliferation of BRAF-mutant melanoma. Moreover, the HDAC6 inhibitor sensitizes BRAF-mutant melanoma cells to vemurafenib-induced cell proliferation inhibition and apoptosis induction, which occur partly through induction of ER stress and inactivation of ERK. Therefore, inhibition of HDAC6 might be a potential strategy for treating melanoma and overcoming resistance to vemurfenib.

\section{Acknowledgements}

The HDAC6 overexpression plasmids were kindly provided by Professor Jun Zhou of College of Life Sciences, Nankai University. This study was supported by Hubei Province Natural Sciences Foundation (no. 2013CFB263).

\section{References}

1. Flaherty KT, Hodi FS and Fisher DE: From genes to drugs: Targeted strategies for melanoma. Nat Rev Cancer 12: 349-361, 2012.

2. Simões MC, Sousa JJ and Pais AA: Skin cancer and new treatment perspectives: A review. Cancer Lett 357: 8-42, 2015.

3. Bollag G, Tsai J, Zhang J, Zhang C, Ibrahim P, Nolop K and Hirth P: Vemurafenib: The first drug approved for BRAF-mutant cancer. Nat Rev Drug Discov 11: 873-886, 2012.

4. Holderfield M, Deuker MM, McCormick F and McMahon M: Targeting RAF kinases for cancer therapy: BRAF-mutated melanoma and beyond. Nat Rev Cancer 14: 455-467, 2014.

5. Strickland LR, Pal HC, Elmets CA and Afaq F: Targeting drivers of melanoma with synthetic small molecules and phytochemicals. Cancer Lett 359: 20-35, 2015.

6. Romano E, Pradervand S, Paillusson A, Weber J, Harshman K, Muehlethaler K, Speiser D, Peters S, Rimoldi D and Michielin O: Identification of multiple mechanisms of resistance to vemurafenib in a patient with $\mathrm{BRAF}^{\mathrm{V} 600 \mathrm{E}}$-mutated cutaneous melanoma successfully rechallenged after progression. Clin Cancer Res 19: 5749-5757, 2013. 
7. Paraiso KH, Haarberg HE, Wood E, Rebecca VW, Chen YA, Xiang Y, Ribas A, Lo RS, Weber JS, Sondak VK, et al: The HSP90 inhibitor XL888 overcomes BRAF inhibitor resistance mediated through diverse mechanisms. Clin Cancer Res 18 2502-2514, 2012.

8. Falkenberg KJ and Johnstone RW: Histone deacetylases and their inhibitors in cancer, neurological diseases and immune disorders. Nat Rev Drug Discov 13: 673-691, 2014.

9. Kaliszczak M, Trousil S, Åberg O, Perumal M, Nguyen QD and Aboagye EO: A novel small molecule hydroxamate preferentially inhibits HDAC6 activity and tumour growth. Br J Cancer 108: 342-350, 2013

10. Liu J, Gu J, Feng Z, Yang Y, Zhu N, Lu W and Qi F: Both HDAC5 and HDAC6 are required for the proliferation and metastasis of melanoma cells. J Transl Med 14: 7, 2016.

11. Woan KV, Lienlaf M, Perez-Villaroel P, Lee C, Cheng F, Knox T, Woods DM, Barrios K, Powers J, Sahakian E, et al: Targeting histone deacetylase 6 mediates a dual anti-melanoma effect: Enhanced antitumor immunity and impaired cell proliferation. Mol Oncol 9: 1447-1457, 2015.

12. Wang L, Xiang S, Williams KA, Dong H, Bai W, Nicosia SV, Khochbin S, Bepler G and Zhang X: Depletion of HDAC6 enhances cisplatin-induced DNA damage and apoptosis in non-small cell lung cancer cells. PLoS One 7: e44265, 2012.

13. Wang Z, Hu P, Tang F, Lian H, Chen X, Zhang Y, He X, Liu W and Xie C: HDAC6 promotes cell proliferation and confers resistance to temozolomide in glioblastoma. Cancer Lett 379: 134-142, 2016

14. Yoshida M, Furumai R, Nishiyama M, Komatsu Y, Nishino N and Horinouchi S: Histone deacetylase as a new target for cancer chemotherapy. Cancer Chemother Pharmacol 48 (Suppl 1): S20-S26, 2001.

15. Krämer OH, Mahboobi S and Sellmer A: Drugging the HDAC6HSP90 interplay in malignant cells. Trends Pharmacol Sci 35: 501-509, 2014

16. Yang Y, Ran J, Liu M, Li D, Li Y, Shi X, Meng D, Pan J, Ou G, Aneja R, et al: CYLD mediates ciliogenesis in multiple organs by deubiquitinating Cep70 and inactivating HDAC6. Cell Res 24 $1342-1353,2014$

17. Santo L, Hideshima T, Kung AL, Tseng JC, Tamang D, Yang M, Jarpe M, van Duzer JH, Mazitschek R, Ogier WC, et al: Preclinical activity, pharmacodynamic, and pharmacokinetic properties of a selective HDAC6 inhibitor, ACY-1215, in combination with bortezomib in multiple myeloma. Blood 119: 2579-2589, 2012

18. Raje N, Vogl DT, Hari PN, et al: ACY-1215, a selective histone deacetylase (HDAC) 6 inhibitor: Interim results of combination therapy with bortezomib in Patients with multiple myeloma (MM). Blood 122: 759, 2013

19. Pandey UB, Nie Z, Batlevi Y, McCray BA, Ritson GP, Nedelsky NB, Schwartz SL, DiProspero NA, Knight MA, Schuldiner O, et al: HDAC6 rescues neurodegeneration and provides an essential link between autophagy and the UPS Nature 447: 859-863, 2007.

20. Lee JY, Koga H, Kawaguchi Y, Tang W, Wong E, Gao YS, Pandey UB, Kaushik S, Tresse E, Lu J, et al: HDAC6 controls autophagosome maturation essential for ubiquitin-selective quality-control autophagy. EMBO J 29: 969-980, 2010.

21. Senft D and Ronai ZA: UPR, autophagy, and mitochondria crosstalk underlies the ER stress response. Trends Biochem Sci 40: 141-148, 2015

22. Beck D, Niessner H, Smalley KS, Flaherty K, Paraiso KH, Busch C, Sinnberg T, Vasseur S, Iovanna JL, Drießen S, et al: Vemurafenib potently induces endoplasmic reticulum stressmediated apoptosis in BRAFV600E melanoma cells. Sci Signal 6: ra7, 2013.

23. Rodvold JJ, Mahadevan NR and Zanetti M: Immune modulation by ER stress and inflammation in the tumor microenvironment. Cancer Lett 380: 227-236, 2016.
24. Rutkowski DT and Kaufman RJ: A trip to the ER: Coping with stress. Trends Cell Biol 14: 20-28, 2004.

25. Oyadomari S and Mori M: Roles of CHOP/GADD153 in endoplasmic reticulum stress. Cell Death Differ 11: 381-389, 2004.

26. Rizos H, Menzies AM, Pupo GM, Carlino MS, Fung C, Hyman J, Haydu LE, Mijatov B, Becker TM, Boyd SC, et al: BRAF inhibitor resistance mechanisms in metastatic melanoma: Spectrum and clinical impact. Clin Cancer Res 20: 1965-1977, 2014.

27. Samatar AA and Poulikakos PI: Targeting RAS-ERK signalling in cancer: Promises and challenges. Nat Rev Drug Discov 13. 928-942, 2014.

28. Tien SC and Chang ZF: Oncogenic Shp2 disturbs microtubule regulation to cause HDAC6-dependent ERK hyperactivation. Oncogene 33: 2938-2946, 2014

29. Ott PA, Hodi FS and Robert C: CTLA-4 and PD-1/PD-L1 blockade: New immunotherapeutic modalities with durable clinical benefit in melanoma patients. Clin Cancer Res 19: 5300-5309, 2013

30. Merelli B, Massi D, Cattaneo L and Mandalà M: Targeting the PD1/PD-L1 axis in melanoma: Biological rationale, clinical challenges and opportunities. Crit Rev Oncol Hematol 89: 140-165, 2014.

31. Tumeh PC, Harview CL, Yearley JH, Shintaku IP, Taylor EJ, Robert L, Chmielowski B, Spasic M, Henry G, Ciobanu V, et al: PD-1 blockade induces responses by inhibiting adaptive immune resistance. Nature 515: 568-571, 2014.

32. Hao M, Song F, Du X, Wang G, Yang Y, Chen K and Yang J: Advances in targeted therapy for unresectable melanoma: New drugs and combinations. Cancer Lett 359: 1-8, 2015.

33. Lienlaf M, Perez-Villarroel P, Knox T, Pabon M, Sahakian E, Powers J, Woan KV, Lee C, Cheng F, Deng S, et al: Essential role of HDAC6 in the regulation of PD-L1 in melanoma. Mol Oncol 10: 735-750, 2016

34. Prahallad A, Sun C, Huang S, Di Nicolantonio F, Salazar R, Zecchin D, Beijersbergen RL, Bardelli A and Bernards R: Unresponsiveness of colon cancer to BRAF(V600E) inhibition through feedback activation of EGFR. Nature 483: 100-103, 2012.

35. Corcoran RB, Ebi H, Turke AB, Coffee EM, Nishino M, Cogdill AP, Brown RD, Della Pelle P, Dias-Santagata D, Hung KE, et al: EGFR-mediated re-activation of MAPK signaling contributes to insensitivity of BRAF mutant colorectal cancers to RAF inhibition with vemurafenib. Cancer Discov 2: 227-235, 2012.

36. Gao YS, Hubbert CC and Yao TP: The microtubule-associated histone deacetylase 6 (HDAC6) regulates epidermal growth factor receptor (EGFR) endocytic trafficking and degradation. J Biol Chem 285: 11219-11226, 2010

37. Deribe YL, Wild P, Chandrashaker A, Curak J, Schmidt MH, Kalaidzidis Y, Milutinovic N, Kratchmarova I, Buerkle L, Fetchko MJ, et al: Regulation of epidermal growth factor receptor trafficking by lysine deacetylase HDAC6. Sci Signal 2: ra84, 2009

38. Wang Z, Hu P, Tang F and Xie C: HDAC6-mediated EGFR stabilization and activation restrict cell response to sorafenib in non-small cell lung cancer cells. Med Oncol 33: 50, 2016.

39. Wang Z, Tang F, Hu P, Wang Y, Gong J, Sun S and Xie C: HDAC6 promotes cell proliferation and confers resistance to gefitinib in lung adenocarcinoma. Oncol Rep 36: 589-597, 2016.

40. Sanduja S, Feng Y, Mathis RA, Sokol ES, Reinhardt F, Halaban R and Gupta PB: AMPK promotes tolerance to Ras pathway inhibition by activating autophagy. Oncogene 35: 5295-5303, 2016 